\title{
High Speed Model Implementation and Inversion Techniques for Ferroelectric and Ferromagnetic Transducers
}

\author{
Thomas R. Braun* and Ralph C. Smith ${ }^{\dagger}$ \\ Center for Research in Scientific Computation \\ Department of Mathematics \\ North Carolina State University, Raleigh, NC 27695
}

\begin{abstract}
Ferroelectric and ferromagnetic materials are employed as both actuators and sensors in a wide variety of applications including fluid pumps, nanopositioning stages, sonar transducers, vibration control, ultrasonic sources, and high-speed milling. They are attractive because the resulting transducers are solid-state and often very compact. However, the coupling of field to mechanical deformation, which makes these materials effective transducers, also introduces hysteresis and time-dependent behaviors that must be accommodated in device designs and models before the full potential of compounds can be realized. In this paper, we present highly efficient modeling techniques to characterize hysteresis and constitutive nonlinearities in ferroelectric and ferromagnetic compounds and model inversion techniques which permit subsequent linear control designs.
\end{abstract}

\section{Ferroelectric and Ferromagnetic Transducer Materials}

Ferroelectric and ferromagnetic materials are increasingly considered as actuators and sensors for aerospace, aeronautic, and industrial automotive applications due to their unique transduction capabilities. Actuator capabilities are derived from the converse effect in which input fields produce deformations in the material whereas the direct effect, comprised of stress-induced fields, provides the materials with sensor capabilities. Ferroelectric materials are presently employed in microphones, accelerometers, fluid pumps, nanopositioning stages, sonar transducers, vibration sensors and actuators, ultrasonic sources, inkjet printers, and camera focusing mechanisms. Ferromagnetic transducers are typically bulkier than their ferroelectric counterparts, due to the circuitry and housing required to produce magnetic fields, but they generally provide greater input forces. They are also presently being considered for a wide variety of applications including torque sensors, high-speed milling, ultrasonic sources, sonar transduction, and vibration sensing and attenuation. Details regarding present and predicted applications can be found in [24].

However, the electromechanical and magnetomechanical coupling mechanisms that endow the compounds with unique transducer capabilities also produce hysteresis and constitutive nonlinearities that must be accommodated in designs before the multifunctional material attributes can be fully realized. For certain applications, these nonlinear effects can be minimized by restricting input field levels; however, this limits the applicability of the materials. For ferroelectric devices, hysteresis can be mitigated through the use of charge or current controlled amplifiers [11-14]. However, this is often significantly more expensive then using voltage controlled amplifiers and current control is ineffective if maintaining a DC bias as required for many positioning mechanisms. A third alternative is to linearize hysteretic devices using feedback control. Whereas effective at lower frequencies, this technique becomes less effective at higher frequencies if sampling rates are limited. Moreover, extensive linearization can limit control authority for prescribed tasks such as vibration attenuation or reference signal tracking.

\footnotetext{
*Email: tbraun@pobox.com; Telephone: 919-854-2746

${ }^{\dagger}$ Email: rsmith@eos.ncsu.edu; Telephone: 919-515-7552
} 
To fully utilize the unique transduction capabilities of ferroelectric and ferromagnetic materials for high performance applications, it is thus necessary to construct models that are appropriate for material characterization, device designs, and control implementations. The objectives dictate that the models quantify hysteresis and constitutive nonlinearities in a manner that is sufficiently accurate to meet design specifications and sufficiently efficient to permit real-time implementation. Moreover, models must be efficiently inverted to provide filters which approximately linearize transducer dynamics for subsequent linear control design.

Several classes of models for ferroelectric and ferromagnetic materials meet these criteria including homogenized energy models $[1,2,24-26,29,30]$, Preisach models $[6,16,17,23,31,34]$, and domain wall models $[5,9,15,28]$. Moreover, inverse models based on these techniques have been experimentally implemented in $[7,8,17-19,31-33]$.

In this paper, we focus on the development of highly efficient implementation and inversion techniques for the homogenized energy model for subsequent use in high speed design and control algorithms. We employ this technique due to its physical basis, which permits correlation of parameters with measured properties of data [24], and it capability to characterize a wide range of physical behaviors including closure of biased minor loops in quasistatic regimes, thermal relaxation or creep, reversible behavior prior to switching, and temperature-dependent dynamics. Details regarding the framework including a discussion of how it compares and contrasts to Preisach models, can be found in [24]. Throughout, the algorithms will be compared using parameters obtained from data fits to PLZT (a ferroelectric) and Terfenol-D (a ferromagnetic). Representative fits are shown in Figure 1.

This work extends previous work in a number of regards. We establish here that the continuity of field-polarization or field-magnetization relations is maintained with approximation but also demonstrate numerical integration or quadrature stepsize requirements to achieve this continuity numerically. This directly impacts subsequent inversion algorithms. We then discuss new quadrature techniques to improve the continuity of approximate relations for improved inversion and control implementation. As an extension to [2], we develop look-up table and rational Chebyshev techniques to improve the implementation for certain applications and we develop implementation techniques to characterize the displacements generated by ferroelectric or ferromagnetic rods. Finally, we design highly efficient algorithms to construct inverse models that incorporate thermal relaxation for subsequent linear control design.

In Section 2 we introduce implementation algorithms for nonlinear and hysteretic field-polarization/fieldmagnetization relations and in Section 3 we employ these relations to construct algorithms for displacements generated by ferroelectric or ferromagnetic rods. The implementation of the inverse model is addressed and numerically demonstrated in Section 4 .

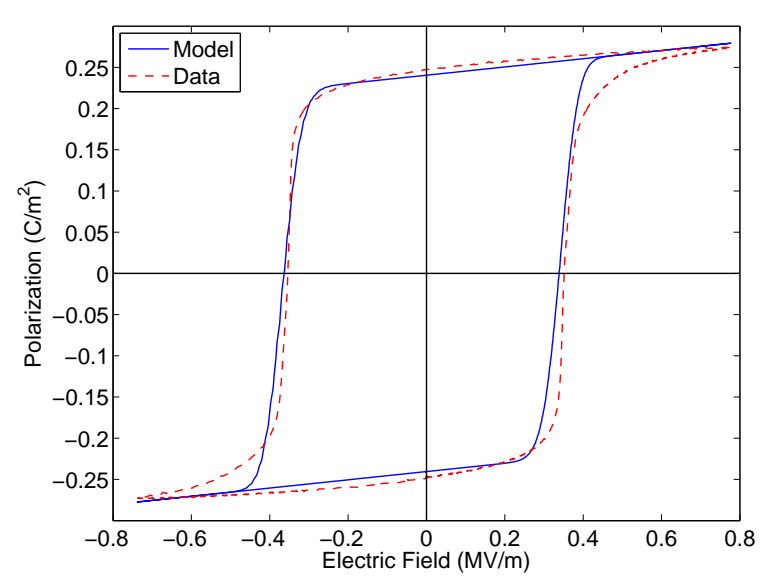

(a)

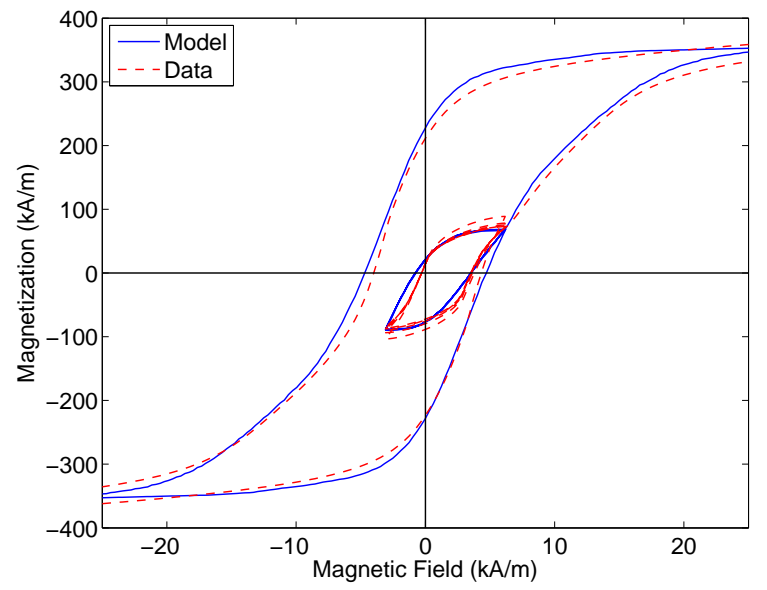

(b)

Figure 1: Example experimental data and model fits for (a) PLZT and (b) Terfenol-D. 


\section{Homogenized Energy Model}

\subsection{Theoretical Development}

We summarize here the homogenized energy model for ferroelectric and ferromagnetic materials [1,24-26, 29,30]. To simplify the discussion we develop it in the context of ferroelectric materials and note that analogous relations hold for ferromagnetic compounds. We note that this is a multiscale approach in which mesoscopic behavior is quantified via energy principles and a macroscopic model is subsequently constructed via stochastic homogenization techniques. The macroscopic ferroelectric model is

$$
P\left(E ; x_{+}\right)=\int_{0}^{\infty} \int_{-\infty}^{\infty} \nu_{c}\left(E_{c}\right) \nu_{I}\left(E_{I}\right)\left[\bar{P}\left(E+E_{I} ; E_{c} ; x_{+}\right)\right] d E_{I} d E_{c}
$$

where $P$ denotes the polarization, $E$ is the electric field, $E_{c}$ denotes the coercive field value at which the dipoles change orientation, $E_{I}$ quantifies the interaction field due to dipole interactions, and $\bar{P}$ is a mesoscale polarization relation. The two model parameters $E_{c}$ and $E_{I}$ are assumed to vary throughout the material according to the densities $\nu_{c}$ and $\nu_{I}$. The densities are constrained by the the physical relations:

1. both $\nu_{c}$ and $\nu_{I}$ are bounded by decaying exponentials,

2. $\nu_{c}$ is strictly positive,

3. $\nu_{I}$ is symmetric about 0 , and

4. $\int_{0}^{\infty} \nu_{c}\left(E_{c}\right) d E c=1, \quad \int_{-\infty}^{\infty} \nu_{I}\left(E_{I}\right) d E_{I}=1$.

The integrals in (1) are solved numerically via quadrature relations, that is,

$$
P\left(E ; x_{+}\right)=\sum_{i=1}^{N_{c}} \sum_{j=1}^{N_{I}} \nu_{c}\left(E_{c}[i]\right) \nu_{I}\left(E_{I}[j]\right) w_{c}[i] w_{I}[j] \bar{P}\left(E+E_{I}[j] ; E_{c}[i] ; x_{+}[i, j]\right)
$$

where $w_{c}$ and $w_{I}$ give the quadrature weights.

The kernel $\bar{P}$ is modeled via energy principles. The mesoscopic Helmholtz energy is taken to be

$$
\psi(P)= \begin{cases}\eta\left(P+P_{R}\right)^{2} / 2, & P \leq-P_{I} \\ \frac{\eta}{2}\left(P_{I}-P_{R}\right)\left(\frac{P^{2}}{P_{I}}-P_{R}\right), & |P|<P_{I} \\ \eta\left(P-P_{R}\right)^{2} / 2, & P \geq P_{I}\end{cases}
$$

where $P_{I}=P_{R}-E_{c} / \eta$ denotes the positive inflection point at which the dipoles switch orientation, $P_{R}$ is the local remanence polarization, and $\eta$ is the reciprocal slope $\frac{\partial E}{\partial P}$. This energy relation is plotted in Figure 2 .

The Gibbs free energy

$$
G=\psi-E P
$$

balances this internal Helmholtz energy with the electrostatic energy; i.e., work performed by the applied external field. As detailed in [24], where the Legendre transform properties of the Gibbs energy are discussed, $G$ is a function of the independent variable $E$ and the dependent variable $P$.

For regimes in which thermal relaxation is negligible, direct minimization of (5) yields the kernel

$$
\bar{P}\left(E+E_{I}, x_{+}\right)=\frac{E+E_{I}}{\eta}+2 P_{R} x_{+}-P_{R}
$$

where $x_{+}=1$ for positively oriented dipoles and $x_{+}=0$ for negatively oriented dipoles. For algorithm development, $x_{+}$is set to 1 whenever $E+E_{I}>E_{c}$ and is set to 0 whenever $E+E_{I}<-E_{c}$. Thermal activation is manifested by dipoles having sufficient thermal energy to switch states before a minima of the Gibbs energy is eliminated. To quantify this, the Gibbs energy is balanced with the relative thermal energy through Boltmann's relation. It is shown in $[24,25,30]$ that the resulting kernel is

$$
\bar{P}=x_{+}\left\langle P_{+}\right\rangle+\left(1-x_{+}\right)\left\langle P_{-}\right\rangle
$$




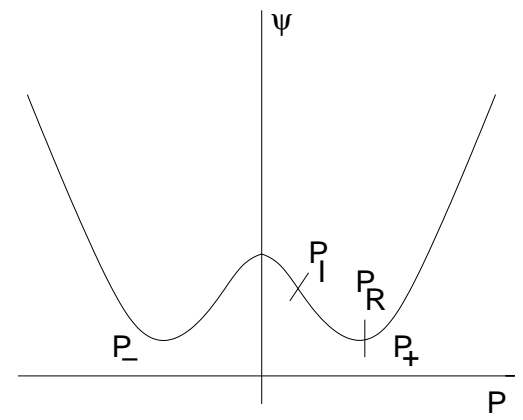

Figure 2: Helmholtz energy function (4)

where $x_{+} \in[0,1]$ denotes the fraction of positively oriented dipoles and

$$
\left\langle P_{+}\right\rangle=\frac{\int_{P_{I}}^{\infty} P \exp \left(\frac{-G\left(E+E_{I}, P\right) V}{k T}\right) d P}{\int_{P_{I}}^{\infty} \exp \left(\frac{-G\left(E+E_{I}, P\right) V}{k T}\right) d P} \quad, \quad\left\langle P_{-}\right\rangle=\frac{\int_{-\infty}^{-P_{I}} P \exp \left(\frac{-G\left(E+E_{I}, P\right) V}{k T}\right) d P}{\int_{-\infty}^{-P_{I}} \exp \left(\frac{-G\left(E+E_{I}, P\right) V}{k T}\right) d P}
$$

are the average polarizations associated with positive and negative dipole orientations. Here $V$ is the mesoscopic volume being modeled, $k$ is Boltmann's constant, and $T$ is the temperature in degrees Kelvin. The evolution of dipole fractions is governed by the differential equation

$$
\dot{x}_{+}=-p_{+-} x_{+}+p_{-+}\left(1-x_{+}\right)
$$

where the likelihoods $p_{+-}$and $p_{-+}$of a dipole switching from positive to negative, or vice-versa, are

$$
p_{+-}=\frac{\exp \left(\frac{-G\left(E+E_{I}, P_{I}\right) V}{k T}\right) d P}{\tau(T) \int_{P_{I}}^{\infty} \exp \left(\frac{-G\left(E+E_{I}, P\right) V}{k T}\right) d P} \quad p_{-+}=\frac{\exp \left(\frac{-G\left(E+E_{I},-P_{I}\right) V}{k T}\right) d P}{\tau(T) \int_{-\infty}^{-P_{I}} \exp \left(\frac{-G\left(E+E_{I}, P\right) V}{k T}\right) d P}
$$

and $\tau$ quantifies the material and temperature-dependent relaxation time. It is noted in [24] that whereas these likelihood relations are commonly employed in practice, they must be treated as approximate in a statistical sense.

\subsection{Implementation Formulation}

The average polarization relations (8) and switching likelihood relations (10) are computationally expensive to compute, especially since they must be computed at each quadrature point and each time step. However, the choice of a piecewise quadratic Helmholtz energy, as opposed to a single fourth-order polynomial or other function, poses several advantages. One advantage is it allows these average polarizations and switching likelihoods to be expressed in a more computationally efficient form. To simplify notation, let $E_{e}=E+E_{I}$ denote the effective field. Consider the integral

$$
\int_{P_{I}}^{\infty} \exp \left(\frac{-G\left(E_{e}, P\right) V}{k T}\right) d P
$$

By completing the square, this is equivalent to

$$
\int_{P_{I}}^{\infty} \exp \left(\frac{-G V}{k T}\right) d P=\sqrt{\frac{\pi k T}{2 V \eta}} \exp \left(\frac{V \eta}{2 k T}\left(\frac{E_{e}^{2}}{\eta^{2}}+2 P_{R} \frac{E_{e}}{\eta}\right)\right) \operatorname{erfc}\left(\frac{V \eta}{2 k T}\left(P_{I}-P_{R}-\frac{E_{e}}{\eta}\right)\right) .
$$

The likelihood of switching from positive to negative can thus be formulated as

$$
p_{+-}=\frac{1}{\tau(T)} \sqrt{\frac{2 V \eta}{\pi k T}} \frac{\exp \left(-\frac{V \eta}{2 k T}\left(P_{I}-P_{R}-\frac{E_{e}}{\eta}\right)^{2}\right)}{\operatorname{erfc}\left(\frac{V \eta}{2 k T}\left(P_{I}-P_{R}-\frac{E_{e}}{\eta}\right)\right)}=\frac{\sqrt{2 V \eta}}{\tau(T) \sqrt{\pi k T} \operatorname{erfcx}\left(\frac{V \eta}{2 k T}\left(P_{I}-P_{R}-\frac{E_{e}}{\eta}\right)\right)} .
$$


Similarly, the fundamental theorem of calculus may be applied to $\int_{P_{I}}^{\infty} P \exp \left(\frac{-G V}{k T}\right) d P$ to obtain

$$
\langle P+\rangle=\frac{\sqrt{2 k T}}{\sqrt{\pi V \eta} \operatorname{erfcx}\left(\frac{V \eta}{2 k T}\left(P_{I}-P_{R}-\frac{E_{e}}{\eta}\right)\right)}+\frac{E_{e}}{\eta}+P_{R} .
$$

Similar approaches may be taken for the other average polarization and switching likelihood. Introducing the change of variables

$$
z_{p}=\sqrt{\frac{V}{2 k T \eta}}\left(-E-E_{I}-E_{c}\right), \quad z_{n}=\sqrt{\frac{V}{2 k T \eta}}\left(E+E_{I}-E_{c}\right)
$$

yields the relations

$$
\begin{array}{ll}
p_{+-}=\frac{1}{\tau(T)} \sqrt{\frac{2 V \eta}{\pi k T}} \frac{1}{\operatorname{erfcx}\left(z_{p}\right)}, & \langle P+\rangle=\sqrt{\frac{2 k T}{\pi V \eta}} \frac{1}{\operatorname{erfcx}\left(z_{p}\right)}+\frac{E+E_{I}}{\eta}+P_{R} . \\
p_{-+}=\frac{1}{\tau(T)} \sqrt{\frac{2 V \eta}{\pi k T}} \frac{1}{\operatorname{erfcx}\left(z_{n}\right)}, & \langle P-\rangle=-\sqrt{\frac{2 k T}{\pi V \eta}} \frac{1}{\operatorname{erfcx}\left(z_{n}\right)}+\frac{E+E_{I}}{\eta}-P_{R} .
\end{array}
$$

Since the scaled complementary error functions appear in both switching likelihoods and average polarizations, the value need only be computed once instead of twice. To this end, define

$$
\text { pos }=\frac{1}{\operatorname{erfcx}\left(z_{p}\right)}, \quad n e g=\frac{1}{\operatorname{erfcx}\left(z_{n}\right)} .
$$

The model can then be expressed as

$$
P\left(E ; x_{+}\right)=\frac{E}{\eta}-P_{R}+\sum_{i=1}^{N_{c}} \sum_{j=1}^{N_{I}} \bar{w}_{c}[i] \bar{w}_{I}[j]\left(x_{+}\left(\text {pos }+n e g+k_{1}\right)-n e g\right)
$$

where

$$
\bar{w}_{c}[i]=\sqrt{2 k T / \pi V \eta} \nu_{c}\left(E_{c}[i]\right) w_{c}[i], \quad \bar{w}_{I}[j]=\nu_{I}\left(E_{I}[j]\right) w_{I}[j], \quad k_{1}=P_{R} \sqrt{2 \pi V \eta / k T} .
$$

We note that the relations

$$
\int_{-\infty}^{\infty} \int_{0}^{\infty} \nu_{c}\left(E_{c}\right) \nu_{I}\left(E_{I}\right) d E_{c} d E_{I}=1, \quad \int_{-\infty}^{\infty} \int_{0}^{\infty} \nu_{c}\left(E_{c}\right) \nu_{I}\left(E_{I}\right) E_{I} d E_{c} d E_{I}=0
$$

were used to simplify the equations. These result directly from the physical constraints (2). Similarly the evolution of dipole fractions is given by

$$
\dot{x}_{+}=\frac{1}{k_{2}}\left(-x_{+}(\text {pos }+n e g)+n e g\right)
$$

where

$$
k_{2}=\tau(T) \sqrt{\frac{\pi k T}{2 V \eta}}
$$

The differential equation (18) may be solved by any number of numerical differential equations routines. For example, using the backward Euler method, $x_{+}$is computed at each time step as

$$
x_{+}(t+\Delta t)=\frac{\frac{k_{2}}{\Delta t} x_{+}(t)+n e g}{\frac{k_{2}}{\Delta t}+n e g+p o s} .
$$

A more accurate, but also slightly more computationally intensive approximation, can be solved by assuming $E(t)$ is constant between $t$ and $t+\Delta t$; that is, approximate $E(t)$ by a step function, as is often the case in 
a digital system. In this case, the differential equation is a linear constant coefficient equation, and can be solved analytically to obtain

$$
x_{+}(t+\Delta t)=\frac{n e g}{n e g+p o s}+\left(x_{+}(t)-\frac{n e g}{n e g+p o s}\right) \exp \left(-\frac{\Delta t}{k_{2}}(n e g+p o s)\right) .
$$

Whereas more accurate, the difference between the solution values provided by this formulation and (19) is typically not significant. For example, Figure 3 shows the results of both methods for an example input and parameter set (in this case, taken to match the ferroelectric material PLZT). As can be seen in the magnified version, the difference between the methods is minimal. For most applications, (19) is considered sufficiently accurate and we employ this approach due to its greater computational simplicity.

The formulations given here can significantly improve the run-time of the algorithm as compared with a more direct implementation of the equations in Section 2.1. However, in many cases better performance is still required. This leaves two options: reduce the number of quadrature points or consider additional approximation strategies.

\subsection{Quadrature and the Continuity of $\mathrm{P}$}

It is observed that the computation time of the model is directly proportional to $N_{c} N_{I}$, the number of quadrature points. One may hope to improve computational performance by reducing the number of quadrature points. However, this exaggerates actual or apparent discontinuities in the output $P$.

By definition of the integral, the model (1) is continuous and differentiable. However, the model with quadrature (3) does not retain this smoothness. Since (3) is composed of finite sums, $P$ is smooth if and only if $\bar{P}$ is smooth for every quadrature point. Clearly for the negligible relaxation case (6), $\bar{P}$ is discontinuous, and its value changes by $2 P_{R}$ at the coercive point $E+E_{I}=E_{c}$. Thus, $P$ contains $N_{c} N_{I}$ finite jumps, each of size $2 P_{R} \nu_{c}\left(E_{c}[i]\right) \nu_{I}\left(E_{I}[j]\right) w_{c}[i] w_{I}[j]$ for $i=1 \ldots N_{c}, j=1 \ldots N_{I}$.

For the relaxation case, it can be shown that $P\left(E ; x_{+}\right)$is a continuous function. However, a plot of $P\left(E ; x_{+}\right)$(or rather $M\left(H ; x_{+}\right)$since the parameters come from a least-squares data fit to a Terfenol-D rod) shows that extremely small stepsizes may be required to resolve this continuity. Figure 4(a) shows a plot of $M\left(H ; x_{+}\right)$for various values of $H$ where the state $x_{+}$was held fixed; i.e., each plotted sample was obtained by inputting the given $H$ to the model with the same value of $x_{+}$. The step between each input value of $\mathrm{H}$ was $1 \mathrm{~A} / \mathrm{m}$, and the function appears to be discontinuous. However, zooming in on one of these regions shows that the function is in fact continuous (see Figures $4(\mathrm{~b})-4(\mathrm{~d})$ ). Note, however, that it took a $\Delta H$

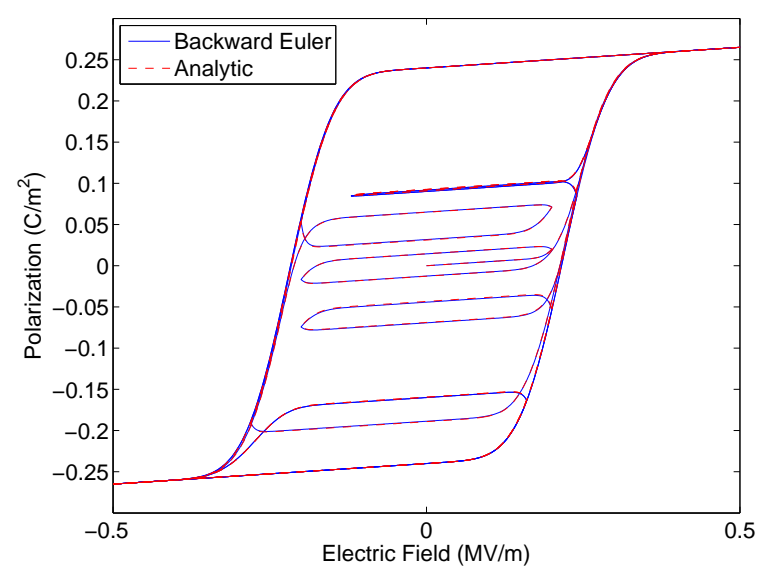

(a)

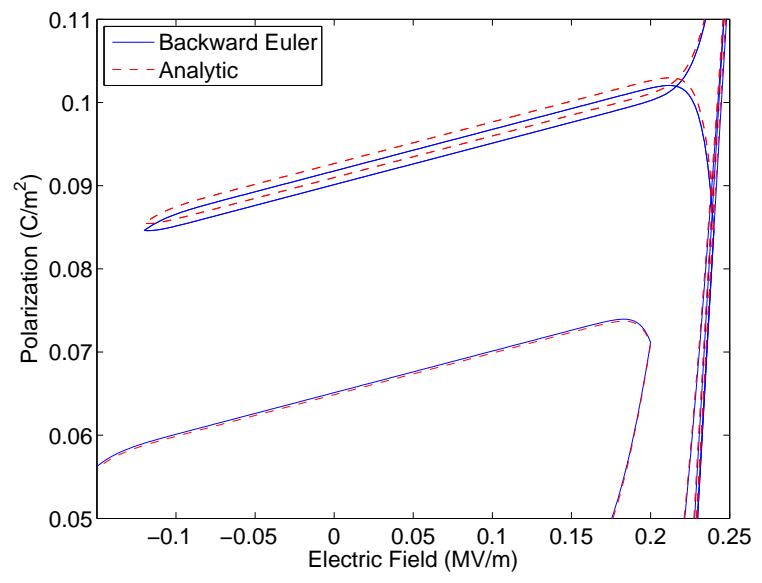

(b)

Figure 3: Comparison between the backward Euler approximation (19) and analytic approximation (20) for an example input, with parameters taken to match PLZT. (a) Entire hysteresis curve, and (b) magnified view. 


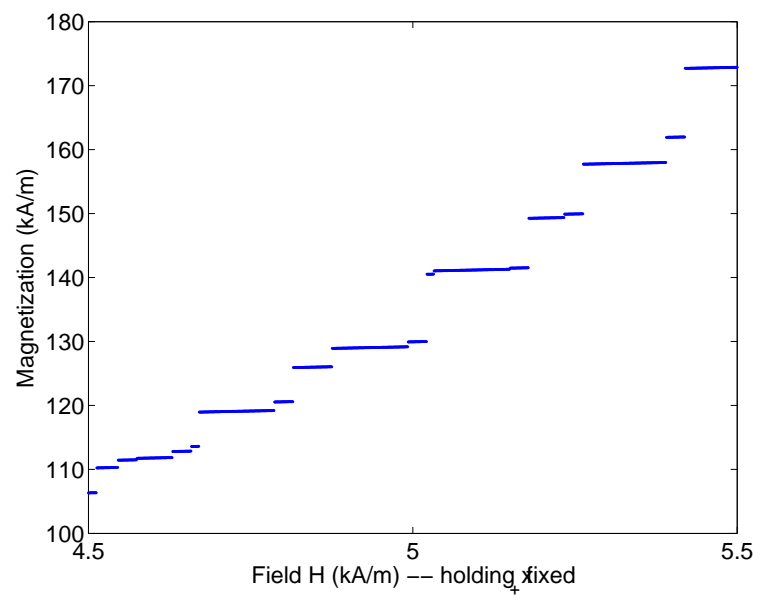

(a) $\Delta H=1$

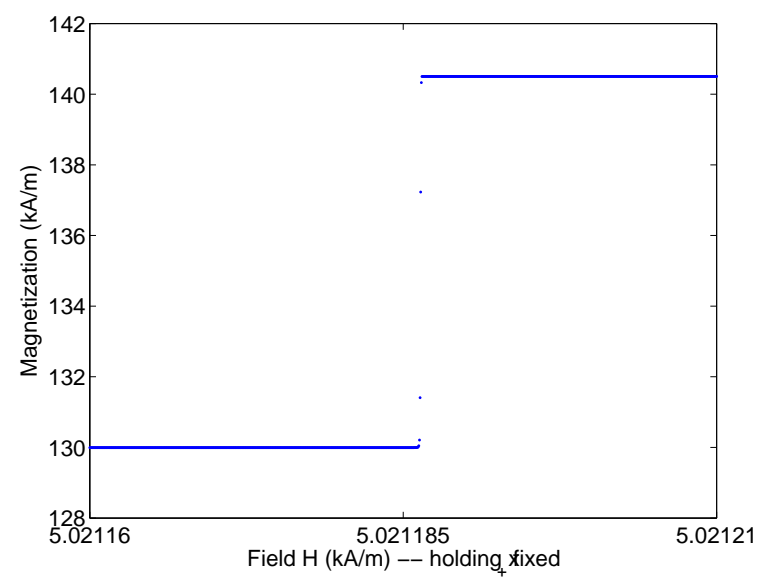

(c) $\Delta H=5 \times 10^{-5}$

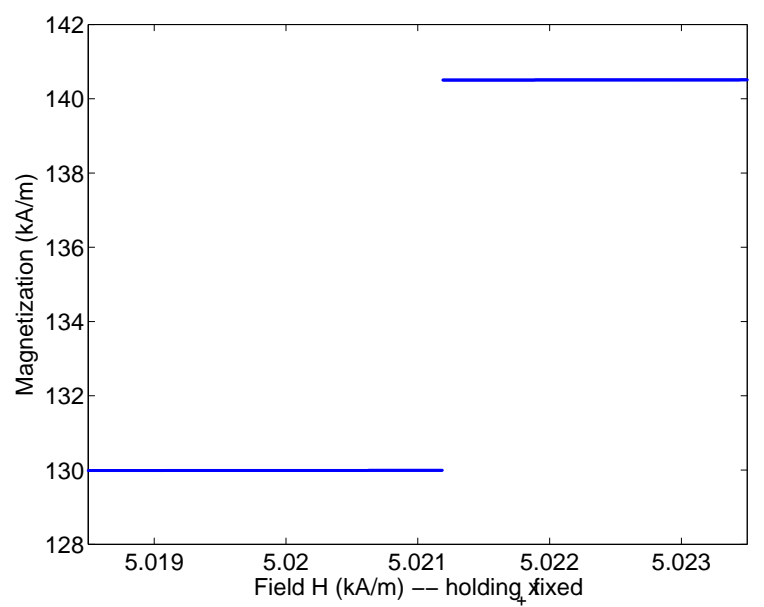

(b) $\Delta H=5 \times 10^{-3}$

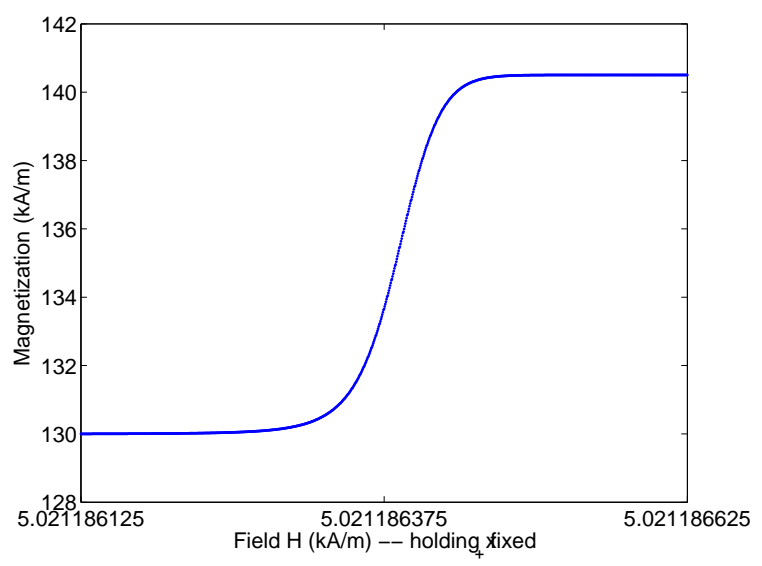

(d) $\Delta H=5 \times 10^{-7}$

Figure 4: Plot of $M\left(H ; x_{+}\right)$for 6 intervals of composite 4-point Gaussian quadrature on each density. For each value of $H, x_{+}$was held fixed; thus the plots represent a single timestep of the model for various input levels. The parameters in these plots were taken from a least-squares fit to Terfenol-D data.

approximately 10 orders of magnitude below $H$ to resolve the function in detail. It is unrealistic to expect such steep slopes to be resolved numerically. In practice, therefore, we must allow for the relaxation model to be discontinuous as well.

Model-based control applications require a smooth model response. As detailed in [24], the relaxation model limits to the negligible relaxation model as $\frac{V}{k T} \rightarrow \infty$. Since, the jump discontinuities in the negligible relaxation model are of height $2 P_{R} \nu_{c}\left(E_{c}[i]\right) \nu_{I}\left(E_{I}[j]\right) w_{c}[i] w_{I}[j]$ for $i=1 \ldots N_{c}, j=1 \ldots N_{I}$, we expect that for large $\frac{V}{k T}$ the "discontinuities" of the relaxation model will also be approximately this height. Thus, the actual or apparent discontinuities may be minimized by minimizing $\nu_{c}\left(E_{c}[i]\right) \nu_{I}\left(E_{I}[j]\right) w_{c}[i] w_{I}[j]$.

One way to increase smoothness is thus to increase the number of quadrature points. If the densities remain unchanged and the number of quadrature points is doubled, then $w_{c}[i] w_{I}[j]$ is halved for all $i, j$. This cuts the size of the jump in half. However, computation time is proportional to the number of quadrature points, so halving the height of the jumps in this way doubles the computation time. This is clearly undesirable. A better situation can be obtained if care is taken on how the quadrature is performed.

Since both $\nu_{c}$ and $\nu_{I}$ are bounded by decaying exponentials, quadrature may be performed on a finite interval and the tails of the distribution need not be considered. For accuracy and efficiency, quadrature is performed by a composite rule; the interval of consideration is divided into several smaller intervals and 
a quadrature rule is applied to each subinterval. Typically, all the subintervals are taken to be equally spaced and this approach was used in the plots given previously. However, equally spaced subintervals are merely a convenience - there is no theoretical reason why one subinterval cannot be bigger than another. In particular, if the region is partitioned such that the integral over each subinterval is equal, then $\nu_{c}\left(E_{c}[i]\right) \nu_{I}\left(E_{I}[j]\right) w_{c}[i] w_{I}[j]$ will be on the same order of magnitude for all $i, j$. (Equality is not obtained because $\nu_{c}$ and $\nu_{I}$ vary on the interval and because for a general quadrature formula not all the weights are equal.) This has the effect of leveling out the jumps, decreasing the big ones while the small ones increase. The result is depicted in Figure 5.

The required number of quadrature points is often determined by accuracy and smoothness requirements. This approach can reduce the number of quadrature points needed for a given smoothness, resulting in significant computational savings.

\subsection{Approximation Methods for Improved Computational Performance}

Reduction of quadrature points alone is not always sufficient to achieve the desired performance of the relaxation model. Significant savings can also be obtained by introducing numerical approximations into the kernel itself. Exploration of the kernel shows that the majority of computation time is spent in the calculation of (16), and we thus focus on methods to approximate pos and neg. This is equivalent to approximating

$$
f(z)=\frac{1}{\operatorname{erfcx}(z)}
$$

since both pos and neg require this function for different values of $z$. The rest of the model is employed in the manner detailed previously.

\subsubsection{Look-up Tables}

The simplest approach conceptually is to store $f(z)$ in a table for values of interest and approximate pos and $n e g$ by looking up the table value nearest $z_{p}$ and $z_{n}$, respectively. At the cost of memory, this reduces the complex erfcx calculation to a simple index calculation and memory access. The indexes into the lookup

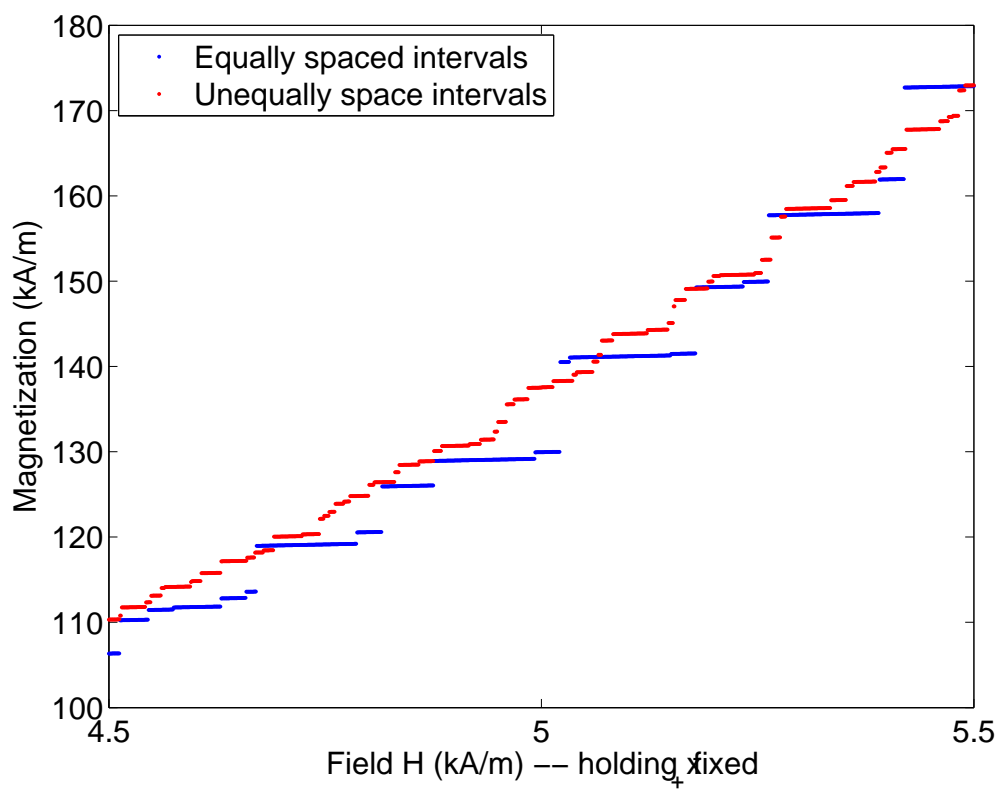

Figure 5: Comparison of $M\left(H ; x_{+}\right)$for 6 intervals of composite 4-point Gaussian quadrature on each distribution. 
table may also be calculated efficiently. Let $h$ be the difference in $z$ between two elements of the table, and let $\ell$ be the $z$ value corresponding to the first (lowest) table entry. Then define

$$
\tilde{E}_{c}[i]=\frac{E_{c}[i]}{h} \sqrt{\frac{V}{2 k T \eta}}-\frac{\ell}{h}, \quad i=1 \ldots N_{c}, \quad \tilde{E}_{I}[j]=\frac{E_{I}[j]}{h} \sqrt{\frac{V}{2 k T \eta}}, \quad j=1 \ldots N_{I}, \quad \tilde{E}=\frac{E}{h} \frac{V}{2 k T \eta} .
$$

Most of these calculations can be performed offline. Only $\tilde{E}$ needs to be computed at each time step and this may be done outside the loops over the quadrature points. Once these values are obtained, the table index is obtained by rounding

$$
\text { pos_index }=-\tilde{E}-\tilde{E}_{I}[j]-\tilde{E}_{c}[i], \quad n e g \_i n d e x=\tilde{E}+\tilde{E}_{I}[j]-\tilde{E}_{c}[i]
$$

to the nearest integer. These values must be checked against the table bounds. An index less than zero signifies a zero should be given instead of a value from the table, whereas an index greater than or equal to the table size implies that the linear approximation should be used instead. Note that the linear approximation for larger $z$ can be adjusted to take the table index (preferably without rounding) instead of the $z_{p}$ or $z_{n}$ as input as well, allowing $\tilde{E}_{c}$ and $\tilde{E}_{I}$ to replace the memory taken by the original quadrature points.

The lookup table approach has several advantages: it is conceptually straightforward, algorithm run-time is independent of table size (within architecture-dependent limits), and approximation error goes to zero as the table size increases. Even relatively small tables yield decent accuracy as illustrated in Table 3 for an example input field. Here it is seen that even 100 table elements produce a root-mean-square error less than approximately $0.25 \%$ of the remanence polarization, and at 10,000 table elements this error was reduced to approximately $0.002 \%$ of the remanence polarization. Note that 10,000 elements is less than $100 \mathrm{~KB}$ of memory when using IEEE double precision floating point numbers. Finally, table values do not need to be recalculated if material properties (i.e., temperature) change. However, there are architectures where this moderate amount of memory is unattainable, or where memory access is too slow. For these cases, we alternatively consider polynomial approximations.

\subsubsection{Rational Chebyshev Approximation}

If the look-up table approximation is infeasible, the function $f(z)$ can instead by approximated by a polynomial or a rational function

$$
R_{m k}(z)=\frac{p_{m}(z)}{q_{k}(z)}
$$

where $p$ is a polynomial of degree $m$ and $q$ is a polynomial of degree $k$. Here $R_{m k}$ is chosen such that

$$
\max w(z)\left|f(z)-R_{m k}(z)\right|
$$

is minimized. This is referred to as a rational Chebyshev or minimax approximation, and is the approach used to implement erfc internally as well. While it can be shown that a unique minimix approximation exists for all $m$ and $k$, computation of these approximations in numerically intensive [22]. However, $f(z)$ does not depend on the material parameters or inputs. Thus, the minimax approximation can be calculated in advance and made available to the code for any choice of parameters. Methods to calculate these approximations using the Remez (also called Remes) algorithm are available in both Mathematica and Maple, alleviating the user from coding minimax algorithms themself.

Like the look-up table, the minimax approximation is applied on a bounded interval. Additionally, the weight function $w(z)$ must be specified. The obvious choices are $w(z)=1$ for absolute error or $w(z)=1 / f(z)$ for relative error. For larger $z$, relative error is a better metric for controlling error. However, for small $z$ calculation of relative error is problematic since $f(z) \longrightarrow 0$. Thus, we let

$$
w(z)=\min \left(1, \frac{\operatorname{erfc}(z)}{\exp \left(-z^{2}\right)}\right)
$$

to use relative error for larger $z$ and absolute error for smaller $z$. The transition from absolute to relative error occurs at $z=0$ and $w(z)$ is continuous. 
We consider two methods to improve the accuracy of the approximation. One approach is to increase $m$ and/or $k$ while the other is to use multiple polynomials over smaller regions instead of a single polynomial over the whole region. Higher degree polynomials increase computational effort in floating point operations whereas adding more polynomials increases conditional statements and control logic. An optimal balance of these is architecture-dependent. As an example, let $f(z)$ be approximated as

$$
f(z) \approx \begin{cases}0 & z<-3 \\ R_{\ell}(z)=\frac{p_{\ell 0}+p_{\ell 1} z+p_{\ell 2} z^{2}+p_{\ell 3} z^{3}}{q_{\ell 0}+q_{\ell 1} z+q_{\ell 2} z^{2}} & -3 \leq z \leq-\frac{1}{2} \\ R_{h}(z)=\frac{p_{h 0}+p_{h 1} z+p_{h 2} z^{2}+p_{h 3} z^{3}}{q_{h 0}+q_{h 1} z+q_{h 2} z^{2}} & -\frac{1}{2}<z \leq 25 \\ m z+b & z \geq 25 .\end{cases}
$$

This employs two minimax approximations with a degree 3 numerator and degree 2 denominator where the regions for each approximation were chosen so that the minimax error (24) is approximately the same. This holds the error (24) below $4 \times 10^{-4}$ for all $z<25$. Since this accuracy is well above the limits imposed by IEEE double precision floating point numbers, further computational savings may be realized by transforming $R_{\ell}(z)$ and $R_{h}(z)$ into continued fraction form, namely

$$
R_{\ell}(z)=a_{\ell 0 c o n s t}+a_{\ell 0 l i n} z+\frac{b_{\ell 1}}{z+a_{\ell 1}+\frac{b_{\ell 2}}{z+a_{\ell 2}}}, \quad R_{h}(z)=a_{h 0 c o n s t}+a_{h 0 l i n} z+\frac{b_{h 1}}{z+a_{h 1}+\frac{b_{h 2}}{z+a_{h 2}}} .
$$

Note that for each of these evaluations only 1 multiply, 2 divides, and 5 additions are required. The values of the relevant coefficients are given in Table 1.

\subsubsection{Method Comparisons}

Neither method is optimal for all situations. However, the look-up table approach is generally the most efficient in terms of run-times and accuracy. Tables 2 and 3 list the run times and errors for an example input. Parameter values were taken to match the ferroelectric material PLZT, and the temperature was assumed to remain constant. It can be seen that either the look-up table or polynomial approximations should almost always be preferred over the exact formulations. As expected, the look-up table approach outperforms the polynomial approach. The run time for various table sizes is not shown because as long as the table was kept small enough to fit in the processor's cache, the run-time was not significantly affected by the size of the table. In cases where the modest memory requirement cannot be achieved, the rational Chebyshev approximation should be used. This takes slightly longer and/or provides less accuracy than the look-up table approximation, but reduces the memory requirement to a handful of values. It is doubtful the memory will ever pose an issue on a conventional processor. Regions where it may prove unattainable are on embedded microprocessors and custom designed field programmable gate arrays (FPGAs) or application specific integrated circuits (ASICs). Rational Chebyshev approximations are a natural choice for these applications.

\section{Displacement Model}

Whereas polarization or inductance/magnetization can sometimes be measured, it is often necessary to formulate the model in terms of displacement instead of polarization. Any such extension requires knowledge of the geometry of the transducer. Rods or beams can be treated with a one-dimensional transducer model, whereas more general shapes require higher-dimension models. As detailed in [24], both nanopositioning stages employed in common atomic force microscope designs and magnetic transducers employed for high speed milling utilize ferroelectric and ferromagnetic rods that are clamped at one end and subject to damped restoring forces at the other. Hence we illustrate the model for a rod of length $\ell$ and cross sectional area $A$ as depicted in Figure 6 . The end mass $m_{\ell}$ with damping $c_{\ell}$ and stiffness $k_{\ell}$ encompass adjacent transducer and plant dynamics. 


\begin{tabular}{ll|ll}
$m$ & 1.771048244745112288944163714 & $b$ & 0.0705327380461303718160865974 \\
\hline$a_{\ell 0 \text { const }}$ & 1.05305341778847673369961876455 & $a_{\ell 0 l i n}$ & 0.141908467579507101523861449079 \\
$a_{\ell 1}$ & 0.652521450575033268529746994429 & $b_{\ell 1}$ & 2.05040334028682659509776710501 \\
$a_{\ell 2}$ & -0.139674180341575164555785667908 & $b_{\ell 2}$ & 2.88659338549683880846513610339 \\
\hline$a_{h 0 \text { const }}$ & 0.0568934411527620915320982226406 & $a_{h 0 l i n}$ & 1.77070610668997459554647064088 \\
$a_{h 1}$ & -12.9327277303294522316468471279 & $b_{h 1}$ & 0.269387658595592656676548244377 \\
$a_{h 2}$ & 16.4152526433497348583844713265 & $b_{h 2}$ & 216.983649137026313567944222991
\end{tabular}

Table 1: Coefficients for the minimax approximation given in (26).

\begin{tabular}{l|rrr} 
& $\begin{array}{c}\text { Intel Xeon } \\
1.7 \mathrm{GHz}\end{array}$ & $\begin{array}{c}\text { Intel Xeon (64 bit) } \\
\text { 3.8 GHz }\end{array}$ & $\begin{array}{c}\text { Intel Pentium-M } \\
2.16 \mathrm{GHz}\end{array}$ \\
& $256 \mathrm{~KB} \mathrm{L2} \mathrm{Cache}$ & MB L2 Cache & 2 MB L2 Cache \\
\hline $\begin{array}{c}\text { Negligible relaxation } \\
\text { algorithm }\end{array}$ & 10,748 & 62,725 & 31,969 \\
$\begin{array}{c}\text { Relaxation - full numerical } \\
\text { precision }\end{array}$ & 429 & 1801 & 1276 \\
$\begin{array}{c}\text { Relaxation - look-up table } \\
\text { approximation } \\
\begin{array}{c}\text { Relaxation - polynomial } \\
\text { approximation }(26)\end{array}\end{array}$ & 5344 & 14,923 & 14,226 \\
& 4997 & 11,674 & 12,177
\end{tabular}

Table 2: Time steps per second computed by each algorithm for three different computer architectures (averaged from 1 million time step). Polynomial approximation was performed using the continued fraction form (27) of the example polynomial given in (26). Look-up tables were computed for $-6 \leq z \leq 25$. In all cases, the model was run with 40 quadrature points for each distribution (1600 total).

\begin{tabular}{l|ll} 
& Peak error (\% of remanence) & RMS error (\% of remanence) \\
\hline Look-up table (100 table elements) & $0.65860 \%$ & $0.25593 \%$ \\
Look-up table (1000 table elements) & $0.063883 \%$ & $0.0055925 \%$ \\
Look-up table (10,000 table elements) & $0.0020362 \%$ & $0.00019354 \%$ \\
Polynomial approximation (26) & $0.5377 \%$ & $0.1647 \%$
\end{tabular}

Table 3: Approximation error for an example input using parameters obtained for PLZT. Polynomial approximation was performed using the continued fraction form (27) of the example polynomial given in (26); look-up tables were computed for $-6 \leq z \leq 25$, and erfcx was implemented directly as exponential and error function evaluations, with the linear approximation utilized for large $z$. Forty quadrature points were given for each distribution (1600 total)

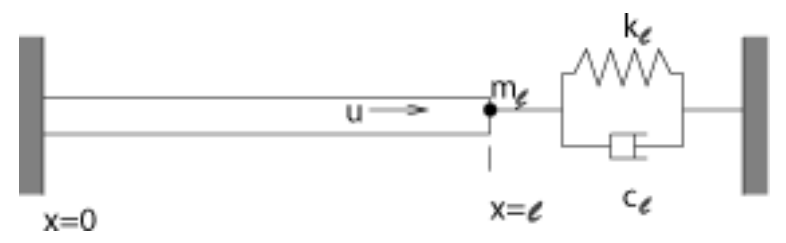

Figure 6: Rod used to construct overall model. The end mass $m_{\ell}$, stiffness $k_{\ell}$ and damping $c_{\ell}$ represent adjacent dynamics of the transducer and plant. 
It is shown in [24] that the constitutive relation for the induced stress is given by

$$
\sigma=Y \varepsilon+C \dot{\varepsilon}-a_{1}\left(P-P_{0}\right)-a_{2}\left(P-P_{0}\right)^{2}
$$

where $Y$ is the Young's modulus of the transducer, $C$ is the Kelvin-Voight damping coefficient, $P_{0}$ is the point the material is poled around, $a_{1}$ and $a_{2}$ are constants of proportionality relating the induced polarization to mechanical force, and $P=P\left(E ; x_{+}\right)$is given in (3).

Due to the distributed nature of the device, partial differential equation models have been developed in $[24,27]$ to characterize the device dynamics. However, for a number of motivating applications, it has been shown that because forces and fields are uniform in space, strains and hence displacements are also uniform thus permitting the use of ordinary differential equation representations. In this case, the strain is given by

$$
\varepsilon(t)=\frac{u(t)}{\ell}
$$

where $u(t)$ denotes the displacement of the rod tip at time $t$.

A force balance yields

$$
m \frac{d^{2} u_{\ell}}{d t^{2}}+c \frac{d u_{\ell}}{d t}+k u_{\ell}=\alpha_{1}\left(P-P_{0}\right)+\alpha_{2}\left(P-P_{0}\right)^{2}
$$

where $\rho$ is the density of the rod and

$$
\begin{gathered}
m=\rho A \ell+m_{\ell}, \quad c=\frac{C A}{\ell}+c_{\ell}, \quad k=\frac{Y A}{\ell}+k_{\ell}, \\
\alpha_{1}=A a_{1}, \quad \alpha_{2}=A a_{2} .
\end{gathered}
$$

Further details as well as models for additional geometries may be found in [24,27].

The solution to this differential equation can be obtained with a variety of numerical methods. However, variable-step methods require that $P$ be known at arbitrary times, which in turn requires that the homogenized energy model be solved at arbitrary times. This is typically not feasible in practice. We thus focus on fixed time-step methods. Of these, the most accurate approximation for a given stepsize may be obtained by assuming that $P$ is fixed between time steps so (30) can be solved analytically. This is an approximation, since thermal effects dictate $P$ will vary with time even if $E$ is constant; however, all other fixed step methods implicitly make similar assumptions and include additional approximation error as well.

The approximation of (30) depends on the relation of $\frac{c^{2}}{m^{2}}$ to $\frac{4 k}{m}$. First, to simplify notation let

$$
v=\frac{a_{1}}{m}\left(P-P_{0}\right)+\frac{a_{2}}{m}\left(P-P_{0}\right)^{2} .
$$

Here $v$ is assumed to be a constant, although the value of the constant changes at each time step. Additionally, let

$$
\tilde{k}=\frac{k}{m}, \quad \tilde{c}=\frac{c}{m} .
$$

If $\tilde{c}^{2}>4 \tilde{k}$ then the solution is given by

$$
u(t)=b_{1} \exp \left(-\frac{1}{2}\left(\tilde{c}+\sqrt{\tilde{c}^{2}-4 \tilde{k}}\right) t\right)+b_{2} \exp \left(-\frac{1}{2}\left(\tilde{c}-\sqrt{\tilde{c}^{2}-4 \tilde{k}}\right) t\right) .
$$

Again, to simplify notation let $z=\sqrt{\tilde{c}^{2}-4 \tilde{k}}$. Letting $u(0)=u_{0}$ and $\dot{u}(0)=u_{1}$ gives

$$
b_{1}=\frac{1}{2}\left(1-\frac{\tilde{c}}{z}\right)\left(u_{0}-\frac{v}{\tilde{k}}\right)-\frac{1}{z} u_{1}, \quad b_{2}=\frac{1}{2}\left(1+\frac{\tilde{c}}{z}\right)\left(u_{0}-\frac{v}{\tilde{k}}\right)+\frac{1}{z} u_{1} .
$$

Substitution into (32) and collection of like terms yields

$$
\begin{aligned}
u(t)= & \frac{1}{2}\left(\left(1-\frac{\tilde{c}}{z}\right) \exp \left(-\frac{1}{2}(\tilde{c}+z) t\right)+\left(1+\frac{\tilde{c}}{z}\right) \exp \left(-\frac{1}{2}(\tilde{c}-z) t\right)\right)\left(u_{0}-\frac{v}{\tilde{k}}\right) \\
& +\frac{1}{z}\left(\exp \left(-\frac{1}{2}(\tilde{c}-z) t\right)-\exp \left(-\frac{1}{2}(\tilde{c}+z) t\right)\right) u_{1}+\frac{v}{\tilde{k}} .
\end{aligned}
$$


If we assume the step $\Delta t$ is fixed, then we can define the six constants

$$
\begin{aligned}
& d_{1}=\frac{1}{2}\left(\left(1-\frac{\tilde{c}}{z}\right) \exp \left(-\frac{1}{2}(\tilde{c}+z) \Delta t\right)+\left(1+\frac{\tilde{c}}{z}\right) \exp \left(-\frac{1}{2}(\tilde{c}-z) \Delta t\right)\right), \quad d_{3}=\frac{1}{k}\left(1-d_{1}\right) \\
& d_{2}=\frac{1}{z}\left(\exp \left(-\frac{1}{2}(\tilde{c}-z) \Delta t\right)-\exp \left(-\frac{1}{2}(\tilde{c}+z) \Delta t\right)\right) \\
& d_{4}=\frac{1}{4}\left(\frac{\tilde{c}^{2}}{z}-z\right)\left(\exp \left(-\frac{1}{2}(\tilde{c}+z) \Delta t\right)-\exp \left(-\frac{1}{2}(\tilde{c}-z) \Delta t\right)\right), \quad d_{6}=-\frac{d_{4}}{\tilde{k}} \\
& d_{5}=\frac{1}{2}\left(\left(1+\frac{\tilde{c}}{z}\right) \exp \left(-\frac{1}{2}(\tilde{c}+z) \Delta t\right)+\left(1-\frac{\tilde{c}}{z}\right) \exp \left(-\frac{1}{2}(\tilde{c}-z) \Delta t\right)\right),
\end{aligned}
$$

In terms of these constants, the solution of the rod model for $\tilde{c}^{2}>4 \tilde{k}$ is approximated by

$$
\begin{aligned}
& u(t+\Delta t)=d_{1} u(t)+d_{2} \dot{u}(t)+d_{3} v(t+\Delta t), \\
& \dot{u}(t+\Delta t)=d_{4} u(t)+d_{5} \dot{u}(t)+d_{6} v(t+\Delta t) .
\end{aligned}
$$

A similar approach can be taken for the other two cases. If $\tilde{c}^{2}=4 \tilde{k}$, we again obtain (36) except with the constants given by

$$
\begin{array}{ll}
d_{1}=\left(1+\frac{\tilde{c}}{2} \Delta t\right) \exp \left(-\frac{\tilde{c}}{2} \Delta t\right), & d_{4}=-k \Delta t \exp \left(-\frac{\tilde{c}}{2} \Delta t\right), \\
d_{2}=\Delta t \exp \left(-\frac{\tilde{c}}{2} \Delta t\right), & d_{5}=\left(1-\frac{\tilde{c}}{2} \Delta t\right) \exp \left(-\frac{\tilde{c}}{2} \Delta t\right), \\
d_{3}=\frac{1}{k}\left(1-d_{1}\right), & d_{6}=-\frac{d_{4}}{\tilde{k}} .
\end{array}
$$

Finally, if $\tilde{c}^{2}<4 \tilde{k}$, the values for the constants are

$$
\begin{aligned}
d_{1} & =\exp \left(-\frac{\tilde{c}}{2} t\right)\left(\cos \left(\frac{z}{2} \Delta t\right)+\frac{\tilde{c}}{z} \sin \left(\frac{z}{2} \Delta t\right)\right), & d_{4} & =-\frac{1}{2}\left(z+\frac{\tilde{c}^{2}}{z}\right) \exp \left(-\frac{\tilde{c}}{2} t\right) \sin \left(\frac{z}{2} \Delta t\right) \\
d_{2} & =\frac{2}{z} \exp \left(-\frac{\tilde{c}}{2} t\right) \sin \left(\frac{z}{2} \Delta t\right), & d_{5} & =\exp \left(-\frac{\tilde{c}}{2} t\right)\left(\cos \left(\frac{z}{2} \Delta t\right)-\frac{\tilde{c}}{z} \sin \left(\frac{z}{2} \Delta t\right)\right) \\
d_{3} & =\frac{1}{k}\left(1-d_{1}\right), & d_{6} & =-\frac{d_{4}}{\tilde{k}}
\end{aligned}
$$

Thus, the rod model costs only six multiplies and four additions per iteration, plus three multiplications and two additions for the calculation of $v$. That is, the rod model computation is essentially free when compared to the computational cost of the homogenized energy model.

\section{Inverse Model}

As discussed in Section 1, one method to accurately control a smart material actuator involves the use of an inverse model or compensator in the manner depicted in Figure 7. Linearization in this manner allows simple control techniques including PI/PID and LQR designs to be utilized over the entire range of the actuator. Given a specified displacement, we wish to determine the field necessary to bring the actuator to that value. Such inverse compensators have previously been explored for the negligible relaxation model in [8,24]. Here we consider a method that works for both the relaxation and negligible relaxation models.

The rod model may be inverted analytically. Solving (36) for $v$ yields

$$
v(t)=\frac{1}{d_{3}} u(t)-\frac{d_{1}}{d_{3}} u(t-\Delta t)-\frac{d_{2}}{d_{3}} \dot{u}(t-\Delta t) .
$$




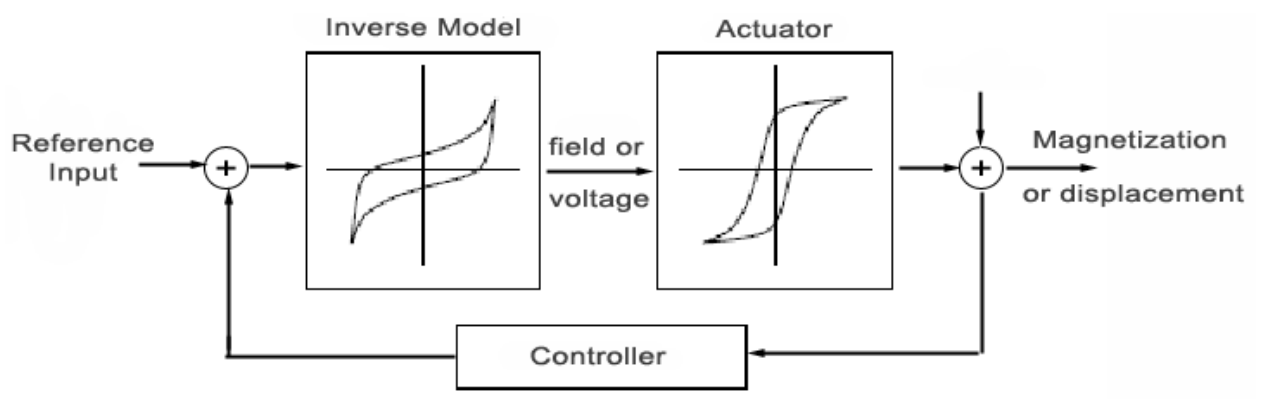

Figure 7: Control configuration utilizing an inverse model and a linear control law.

Once $v$ has been determined, the required polarization can be determined from the quadratic formula, namely

$$
\widehat{P}(t)=P_{0}-\frac{\alpha_{1}}{2 \alpha_{2}} \pm \sqrt{\left(\frac{\alpha_{1}}{2 \alpha_{2}}\right)^{2}+\frac{m v(t)}{a_{2}}}
$$

as long as $\alpha_{2} \neq 0$. If $\alpha_{2}$ is 0 , the equation is linear and the solution is

$$
\widehat{P}(t)=P_{0}+\frac{m}{\alpha_{1}} v(t) .
$$

Note that for $\alpha_{2} \neq 0$, either polarization value is acceptable, as both give equivalent displacements. However, to minimize the effects of modeling error, a single branch should always be utilized.

A definition of the inverse homogenized energy model is the following: given any valid state $x_{+}$and any specified $\widehat{P}$ within the operating range of the material, determine $E$ such that $P=\widehat{P}$. Unlike the rod model, however, it is not feasible to invert (3) analytically. Thus, we reformulate the problem as numerical root finding, namely determining the value $E$ such that for a given $x_{+}$and $\widehat{P}$,

$$
P\left(E ; x_{+}\right)-\widehat{P}=0 .
$$

We note that this function is monotone in $E$ (holding $x_{+}$fixed). However, as discussed in Section 2.3, the function may contain a finite number of simple jumps.

\subsection{Discontinuous Root Finding}

If (42) was continuously differentiable, the secant method would provide nearly optimal convergence to the solution in terms of function evaluations [10]. However, for a discontinuous function it may not converge. The bisection method will converge, but often this convergence is slow. Methods exist which combine bisection with interpolation to attempt to improve this rate; see [3] for example. However, we observe better performance by exploiting the monotone nature of the function to directly improve the convergence of the bisection method.

The bisection method, or any other method which utilizes it, requires both that the root be bounded and that the function change signs within the bounds. For a monotone function, these requirements are the same. Theoretical bounds on $E$ may be computed by considering the case when all dipoles have switched. In these cases (all dipoles positive or all negative), $E$ can be determined analytically. The required value of $E$ is by necessity between these extreme cases, namely

$$
\eta\left(\widehat{P}-P_{R}\right) \leq E \leq \eta\left(\widehat{P}+P_{R}\right) .
$$

However, direct application of the bisection method with these bounds yields slow convergence because the bounds are large.

Instead, we employ the secant method as a way to calculate tighter bounds. The secant method does not require bounds but instead uses approximate derivatives to converge to the root. Since the function is 
not smooth, this may not converge. However, the monotone nature of the function dictates that the secant method will always step in the correct direction. Thus, on each iteration either the secant method does not step far enough, in which case a better approximation to $E$ is obtained, or it steps too far. In this latter case, the iterations have crossed over the root value and provide a bound on the root. We therefore apply the secant method to (42) first. If there is sufficient relaxation or the value $\widehat{P}$ does not lie near any discontinuities, we obtain rapid convergence. If the secant method fails to converge, the secant iterations themselves are utilized to determine the bound on the root which can then be given to bisection to find the actual root. These bounds are typically much tighter than (43), which improves the convergence of the bisection method. For the purposes of our algorithm, failure to converge in the secant method is defined as

$$
\left|P\left(E_{i} ; x_{+}\right)-\widehat{P}\right| \geq\left|P\left(E_{i-2} ; x_{+}\right)-\widehat{P}\right|, \quad \text { for all } i \geq 3
$$

where $E_{i}$ is the value of $E$ determined by the ith iteration of the secant method. Such a definition is a heuristic to detect failure quickly without penalizing a single poor step.

The secant method requires an initial approximation to the derivative to compute its first iteration. One such approximation is $1 / \eta$ which is the derivative if no dipoles are switching. The actual derivative may be much larger than this, but will never be smaller. Another approximation involves using the previous two time steps of the model in a manner analogous to how the secant method itself approximates derivatives. This approximation is simply $\Delta P / \Delta E$, where $\Delta P$ is the difference in $P\left(E ; x_{+}\right)$for the previous two time steps and $\Delta E$ is the corresponding differences in $E$. The latter approximation is deemed more accurate and is utilized when the previous two time steps are known. When this is not the case, $1 / \eta$ is utilized as the initial derivative. The computed derivative value should also be checked against this lower limit on each iteration of the secant method, to trap some errors that may arise with the subtraction of nearly equal numbers.

\subsection{Validation and Performance}

The performance of the inverse model is largely dependent on the material parameters being utilized. More relaxation, smaller variances in $\nu_{c}$ and $\nu_{I}$, and slowly changing reference inputs generally yield better performance than small relaxation, largely varying distributions, or rapidly varying inputs. As an example, consider the inverse model for the stepped polarization shown in Figure 8(a). The parameters are specified as those estimated for PLZT to obtain the model fit in Figure 1(a). Ten intervals with 4-point Gaussian quadrature are utilized for each distribution and the time step is $\Delta t=0.001$. As can be seen, the inverse model accounts for the relaxation in the device, decreasing the electric field as appropriate to hold the polarization constant. The error between the specified and actual values is small - five orders of magnitude below the remanence polarization.

The results in Figure 8 were computed with a small tolerance for the secant and bisection methods. Accuracy and effort for various tolerance levels are compiled in Table 4. Note that the algorithm makes efficient use of computation; reasonable accuracy levels may be obtained with only 2 function evaluations (evaluations of the forward homogenized energy model) per iteration on average, or 5 in the worst case. However, this efficiency is dependent on the input. Figure 9(a) shows an input polarization which changes at roughly an order of magnitude higher rate. Using the same material parameters and time step on this input yields the results in Figure 9(b) and Table 5. The same accuracy level as before requires almost 5 function evaluations on average and 16 in the worst case. While increased, this is still easily attainable. The level of effort also depends on the material being modeled. Scaling Figure 9(a) to an appropriate level and applying parameters for a Terfenol-D rod yields the results in Figure 10 and Table 6. The Terfenol-D parameters require greater work for the same level of accuracy, but relatively speaking they are able to achieve a greater accuracy than that achieved in the PLZT case.

Finally, we note that these observations apply directly to the case when displacement is utilized as input. Figure 11 and Table 7 show the accuracy and level of effort for the Terfenol-D parameters with strain (displacement) as an input. The tolerance for the root finding algorithm was also specified in terms of displacement in this case, with $P\left(E ; x_{+}\right)$input to the rod model on each iteration of secant or bisection to determine the error. We note that the inverse compensator provides an accurate way to control the device while maintaining a reasonable number of computations. 


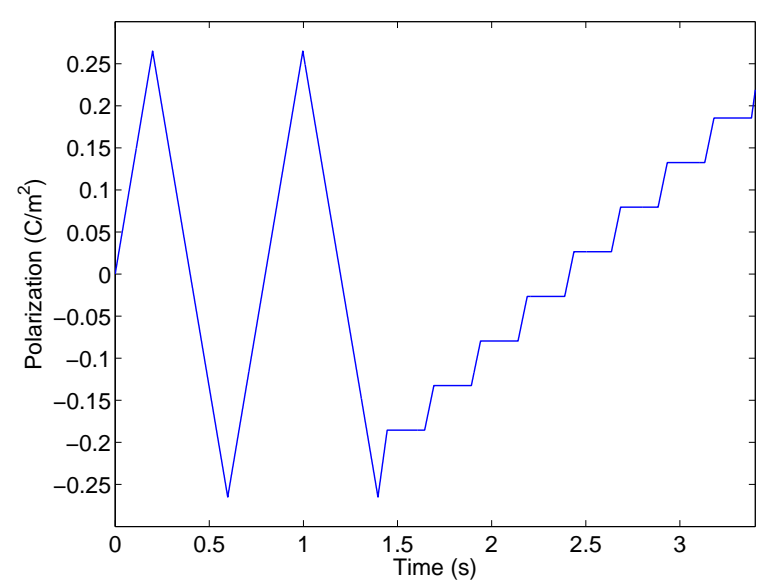

(a)

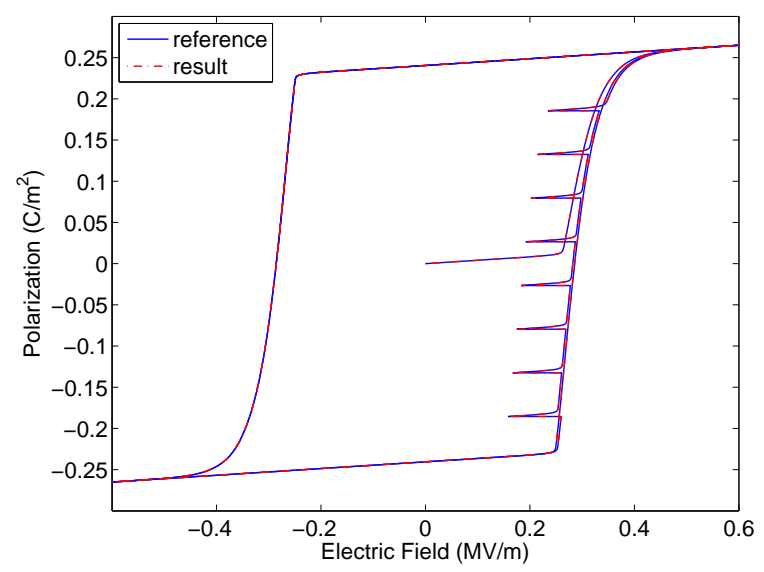

(c)

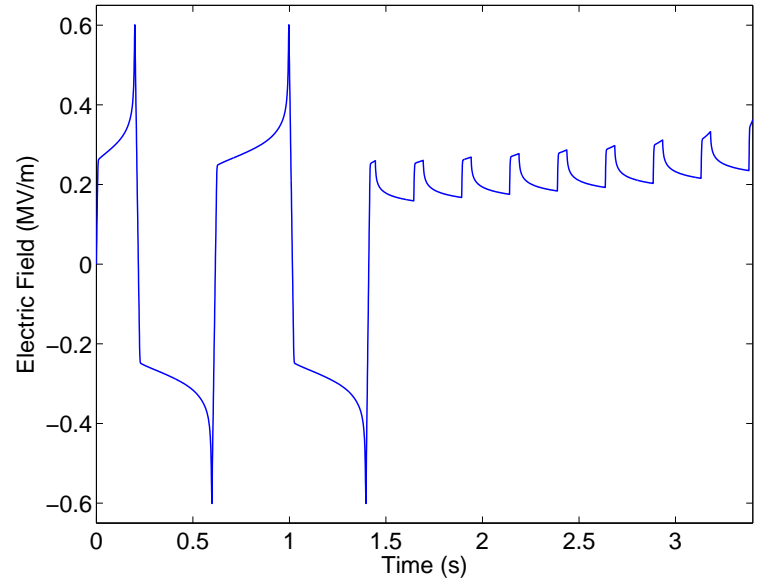

(b)

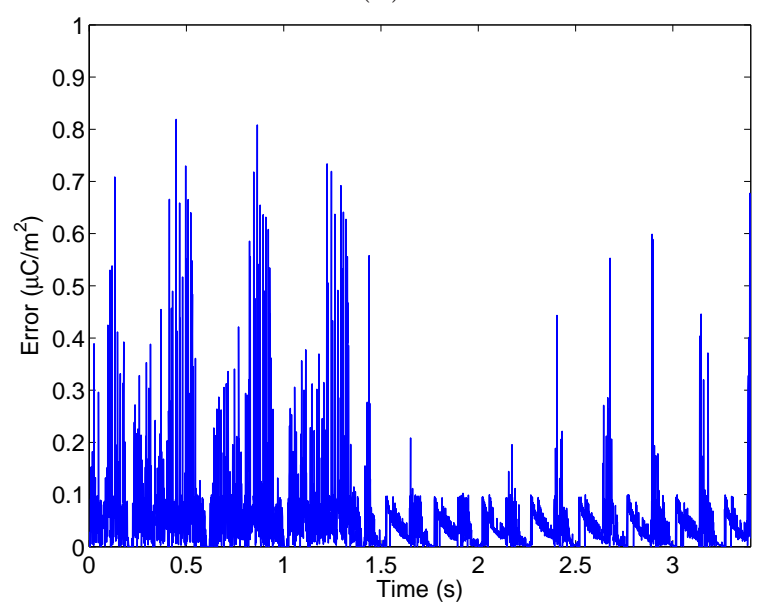

(d)

Figure 8: (a) Reference polarization and (b) electric field $E$ given by the inverse model with parameters estimated for PLZT. (c) Comparison of the resulting polarization $P$ and the reference polarization $\widehat{P}$. (d) Absolute error $|\widehat{P}-P|$.

\begin{tabular}{lll} 
Tolerance & Function Evaluations (Average) & Function Evaluations (Maximum) \\
\hline$P_{R} \times 10^{-2}=0.0024$ & 1.0855 & 3 \\
$P_{R} \times 10^{-3}=0.00024$ & 1.2667 & 4 \\
$P_{R} \times 10^{-4}=2.4 \times 10^{-5}$ & 2.0157 & 5 \\
$P_{R} \times 10^{-5}=2.4 \times 10^{-6}$ & 3.5423 & 9 \\
$P_{R} \times 10^{-6}=2.4 \times 10^{-7}$ & $4.8066 \dagger$ & user-defined
\end{tabular}

Table 4: Effort to compute the inverse model in terms of average and maximum number of function evaluations for various error tolerances, using parameters for PLZT and the input given in Figure 8(a). $\dagger$ denotes that due to discontinuities, some time steps did not converge within the specified tolerance, and only those that did are averaged. In these cases, the maximum number of function evaluations was the user-specified maximum. 


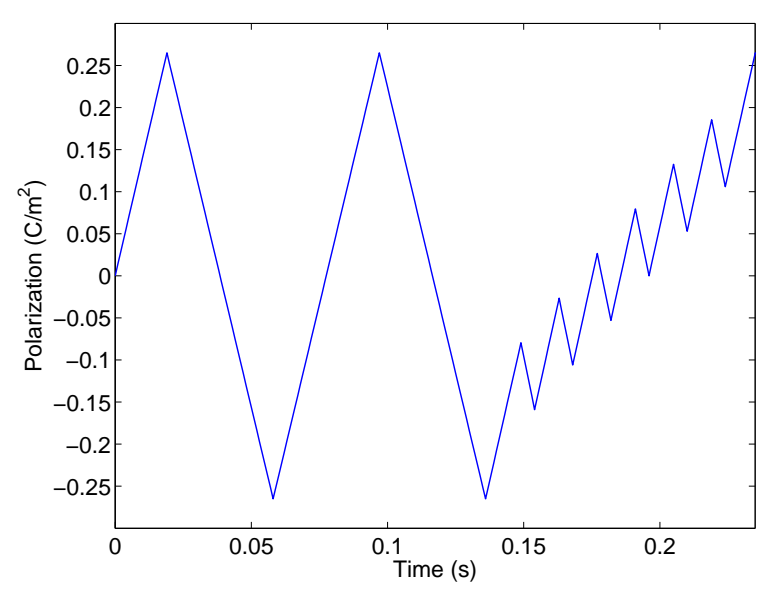

(a)

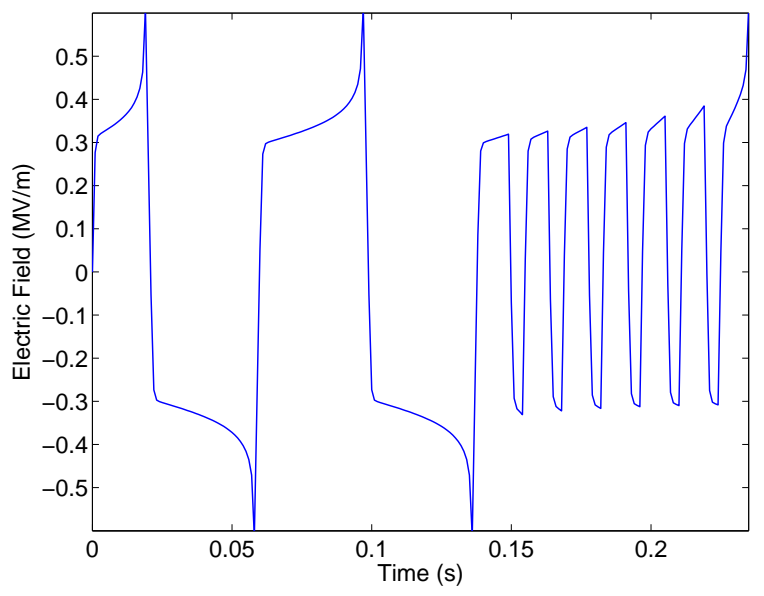

(b)

Figure 9: (a) Reference polarization and (b) electric field $E$ given by the inverse model with parameters estimated for PLZT.

\begin{tabular}{lll} 
Tolerance & Function Evaluations (Average) & Function Evaluations (Maximum) \\
\hline$P_{R} \times 10^{-2}=0.0024$ & 2.8776 & 10 \\
$P_{R} \times 10^{-3}=0.00024$ & 3.9286 & 13 \\
$P_{R} \times 10^{-4}=2.4 \times 10^{-5}$ & 4.8027 & 16 \\
$P_{R} \times 10^{-5}=2.4 \times 10^{-6}$ & $7.9252 \dagger$ & user-defined \\
$P_{R} \times 10^{-6}=2.4 \times 10^{-7}$ & $9.3187 \dagger$ & user-defined
\end{tabular}

Table 5: Effort to compute the inverse model in terms of average and maximum number of function evaluations for various error tolerances, using parameters for PLZT and the input given in Figure 9(a). $\dagger$ denotes that due to discontinuities, some time steps did not converge within the specified tolerance, and only those that did are averaged. In these cases, the maximum number of function evaluations was the user-specified maximum.

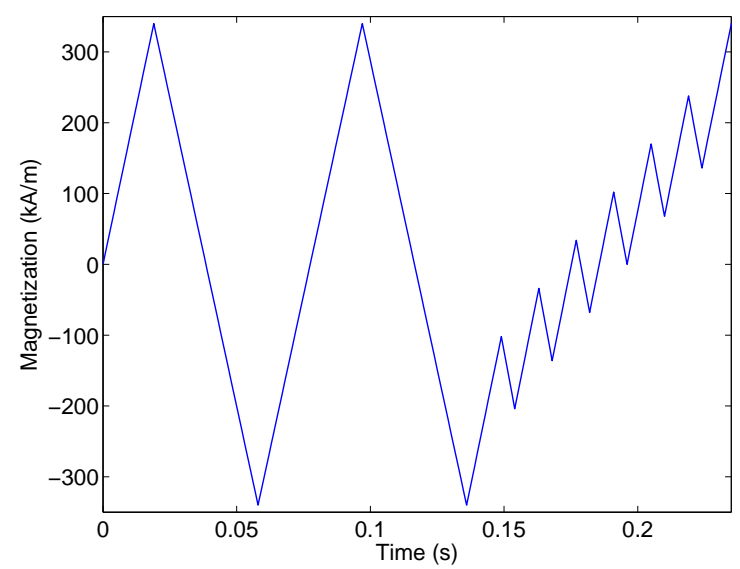

(a)

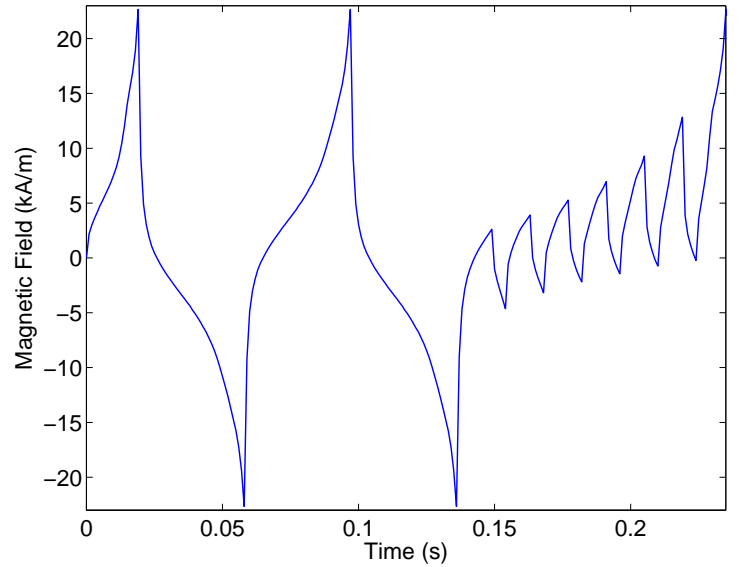

(b)

Figure 10: (a) Reference magnetization and (b) magnetic field $H$ given by the inverse model with parameters estimated for Terfenol-D. 


\begin{tabular}{lll} 
Tolerance & Function Evaluations (Average) & Function Evaluations (Maximum) \\
\hline$M_{R} \times 10^{-2}=3400$ & 2.8673 & 9 \\
$M_{R} \times 10^{-3}=340$ & 5.4354 & 16 \\
$M_{R} \times 10^{-4}=34.0$ & 10.7143 & 22 \\
$M_{R} \times 10^{-5}=3.40$ & 14.2993 & 25 \\
$M_{R} \times 10^{-6}=0.340$ & 17.2279 & 29
\end{tabular}

Table 6: Effort to compute the inverse model in terms of average and maximum number of function evaluations for various error tolerances, using parameters for Terfenol-D and the input given in Figure 10(a).

\section{Concluding Remarks}

In this paper, we have provided a computationally efficient formulation of the homogenized energy model suitable for use in nonlinear model-based control designs, parameter estimation routines, and inversecompensator based-control for ferroelectric and ferromagnetic actuators. We have also demonstrated that the inverse model accurately and efficiently compensates for ferroelectric and ferromagnetic hysteresis in an open-loop of feedforward manner without requiring any a priori knowledge of the reference input. The re-

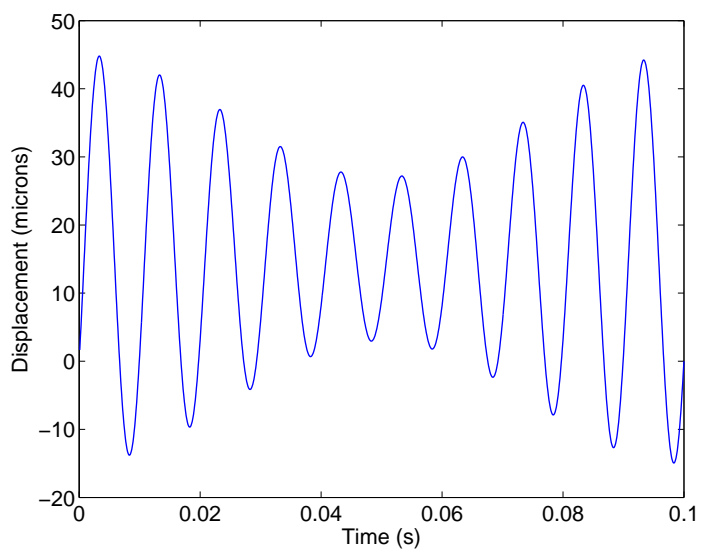

(a)

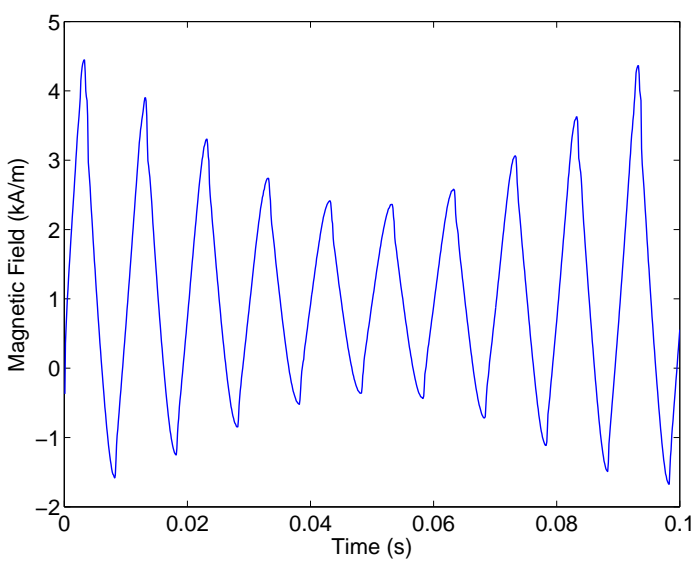

(b)

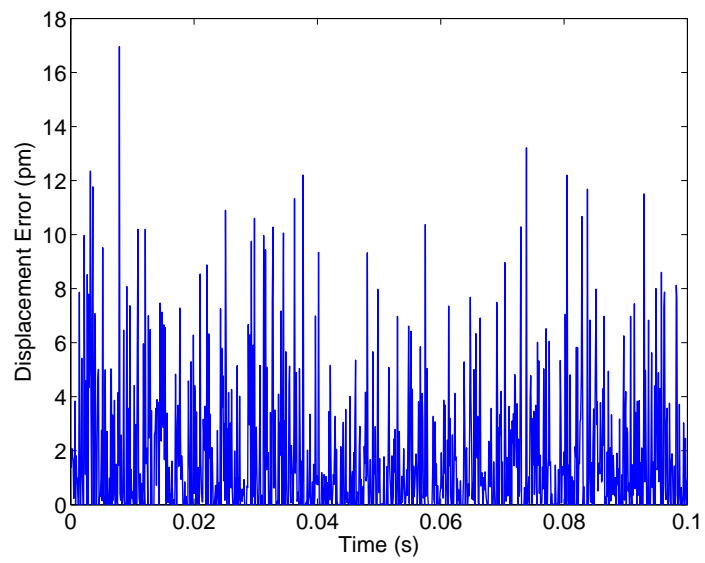

(c)

Figure 11: Results for the combined inverse model with parameters estimated for a Terfenol-D rod. (a) Specified displacement $\widehat{u}$ and (b) magnetic field $H$ provided by the inverse algorithms. (c) Absolute error $|\widehat{u}-u|$ for displacement $u$ given by the rod model. 


\begin{tabular}{ccc}
$\begin{array}{c}\text { Error } \\
\text { tolerance }\end{array}$ & $\begin{array}{c}\text { Average } \\
\text { model evaluations }\end{array}$ & $\begin{array}{c}\text { Maximum } \\
\text { model evaluations }\end{array}$ \\
\hline 1 micron & 2.1240 & 8 \\
$100 \mathrm{~nm}$ & 2.2880 & 9 \\
$10 \mathrm{~nm}$ & 6.5170 & 19 \\
$1 \mathrm{~nm}$ & 11.6500 & 22 \\
$100 \mathrm{pm}$ & 14.7040 & 25 \\
$10 \mathrm{pm}$ & 17.6930 & 29
\end{tabular}

Table 7: Effort to compute the inverse model in terms of average and maximum number of function evaluations utilizing parameters estimated for a Terfenol-D rod and the reference displacement given in Figure 11(a).

sulting inverse algorithms are capable of achieving reasonable accuracy at speeds of 500 - 3000 timesteps per second on a conventional processor, depending on the number of quadrature points and material parameters. With a resolution of 10 timesteps per cycle, this would allow periodic inputs at the rate of $50-300 \mathrm{~Hz}$. Multiprocessor and/or custom integrated circuit (FPGA/ASIC) implementations should allow this rate to be greatly increased and are currently under investigation.

\section{ACKNOWLEDGMENTS}

This research was supported by a United States Department of Education GAANN fellowship and the Air Force Office of Scientific Research under the grant AFOSR-FA9550-04-1-0203.

\section{References}

[1] T.R. Braun, R.C. Smith, and M.J. Dapino, "Experimental validation of a homogenized energy model for magnetic after-effects," Applied Physics Letters, 88(12), 2006.

[2] T.R. Braun and R.C. Smith, "Efficient Implementation Algorithms for Homogenized Energy Models," Continuum Mechanics and Thermodynamics, 18(3-4), pp. 137-155, 2006.

[3] R.P. Brent, Algorithms for Minimization without Derivatives, Prentice-Hall, Englewood Cliffs, N.J., 1973.

[4] W.J. Cody, "Rational chebyshev approximations for the error function," Mathematics of Computation, 23(107), pp. 631-637, 1969.

[5] M.J. Dapino, R.C. Smith, L.E. Faidley, and A.B. Flatau, "A coupled structure-magnetic model for magnetostrictive transducers," Journal of Intelligent Material Systems and Structures, 11(2), pp. 134152,2000 .

[6] P. Ge and M. Jouaneh, "Modeling hysteresis in piezoceramic actuators," Precision Engineering, 17, pp. 211-221, 1995.

[7] P. Ge and M. Jouaneh, "Tracking control of a piezoceramic actuator," IEEE Transactions on Control Systems Technology, 4(3), pp. 206-209, 1996.

[8] A.G. Hatch, R.C. Smith, T. De, and M.V. Salapaka, "Construction and experimental implementation of a model-based inverse filter to attenuate hysteresis in ferroelectric transducers," IEEE Transactions on Control Systems Technology, 14(6), pp. 1058-1069, 2006.

[9] D.C. Jiles and D.L. Atherton, "Theory of ferromagnetic hysteresis", Magnetism and Magnetic Materials, 61, pp. 1265-1281, 1984. 
[10] C.T. Kelley, Iterative Methods for Linear and Nonlinear Equations, SIAM, Philadelphia, 1995.

[11] J.A. Main and E. Garcia, "Design impact of piezoelectric actuator nonlinearities," Journal of Guidance, Control and Dynamics, 20(2), pp. 327-332, 1997.

[12] J.A. Main and E. Garcia, "Piezoelectric stack actuators and control system design: strategies and pitfalls," Journal of Guidance, Control, and Dynamics, 20(3), pp. 479-485, 1997.

[13] J.A. Main, E. Garcia and D.V. Newton, "Precison position control of piezoelectric actuatorss using charge feedback," Journal of Guidance, Control, and Dynamics, 18(5), pp. 1068-73, 1995.

[14] J.A. Main, D. Newton, L. Massengil and E. Garcia, "Efficient power amplifiers for piezoelectric applications," Smart Structures and Materials, 5(6), pp. 766-775, 1996.

[15] J.E. Massad and R.C. Smith, "A domain wall model for hysteresis in ferroelastic materials," Journal of Intelligent Material Systems and Structures, 14(7), pp. 455-471, 2003.

[16] I.D. Mayergoyz, Mathematical Models of Hysteresis, Springer-Verlag, New York, 1991.

[17] S. Mittal and C-H. Meng, "Hysteresis compensation in electromagnetic actuators through Preisach model inversion," IEEE/ASME Transactions on Mechatronics, 5(4), pp. 394-409, 2000.

[18] J.M. Nealis and R.C. Smith, "H $\mathcal{H}_{\infty}$ control design for a magnetostrictive transducer," Proceedings of the 42nd IEEE Conference on Decision and Control, pp. 1801-1806, 2003.

[19] J.M. Nealis and R.C. Smith, "Model-based robust control design for a magnetostrictive transducer operating in hysterestic and nonlinear regimes," IEEE Transactions on Control Systems Technology, 15(1), pp. 22-39, 2007.

[20] W.S. Oates, P. Evans, R.C. Smith, and M.J. Dapino, "Experimental implementation of a nonlinear control method for magnetostrictive transducers," Proceedings of the 46th IEEE conference on Decision and Control, to appear.

[21] W.H. Press, S.A. Teukolsky, W.T. Vetterline, B.P. Flanner, Numerical Recipes in C, 2nd Edition, Cambridge University Press, New York, 1992.

[22] A. Ralston, "Rational Chebyshev approximation", Mathematical Methods for Digital Computers, Vol. 2, John Wiley and Sons, New York, pp. 268-284, 1967.

[23] G. Robert, D. Damjanovic and N. Setter, "Preisach modeling of piezoelectric nonlinearity in ferroelectric ceramics," Journal of Applied Physics, 89(9), pp. 5067-5074, 2001.

[24] R.C. Smith, Smart Material Systems: Model Development, SIAM, Philadelphia, 2005.

[25] R.C. Smith, M.J. Dapino, T.R. Braun and A.P. Mortensen, "A Homogenized energy framework for ferromagnetic hysteresis," IEEE Transactions on Magnetics, 42(7), pp. 1474-1769, 2006.

[26] R.C. Smith, M.J. Dapino and S. Seelecke, "A free energy model for hysteresis in magnetostrictive transducers," Journal of Applied Physics, 93(1), pp.458-466, 2003.

[27] R.C. Smith, A.G. Hatch, T. De, M.V. Salapaka, R.C.H. del Rosario, and J.K. Raye, "Model Development for Atomic Force Microscope Stage Mechanisms," SIAM Journal on Applied Mathematics, 66(6), pp. 1998-2026, 2006.

[28] R.C. Smith and J.E. Massad, "A unified methodology for modeling hysteresis in ferroic materials," Proceedings of the 2001 ASME Design Engineering Technical Conferences and Computer and Information in Engineering Conference, Vol 6, Pt B, pp. 1389-1398, 2001.

[29] R.C. Smith, S. Seelecke, M.J. Dapino and Z. Ounaies, "A unified framework for modeling hysteresis in ferroic materials," Journal of the Mechanics and Physics of Solids, 54(1), pp. 46-85, 2006. 
[30] R.C. Smith, S. Seelecke, Z. Ounaies and J. Smith, "A free energy model for hysteresis in ferroelectric materials," Journal of Intelligent Material Systems and Structures, 14(11), pp. 719-739, 2003.

[31] G. Song, J. Zhao, X. Zhou and J.A. Abreu-Garci, "Tracking control of a piezoceramic actuator with hysteresis compensation using inverse Preisach model,", IEEE/ASME Transactions on Mechatronics, 10(2), pp. 198-209, 2005.

[32] X. Tan and J.S. Baras, "Modeling and control of hysteresis in magnetostrictive actuators," Automatica, 40(9), pp. 1469-1480, 2004.

[33] X. Tan, R. Venkataraman and P.S. Krishnaprasad, "Control of hysteresis: Theory and experimental results," Smart Structures and Materials 2001, Proceedings of the SPIE, Volume 4326, pp. 101-112, 2001.

[34] G. Webb, A. Kurdila and D.C. Lagoudas, "Adaptive hysteresis model for model reference control with actuator hysteresis," Journal of Guidance, Control and Dynamics, 23(3), pp. 459-465, 2000. 